\title{
Aerodynamic analysis and elastic suppression of refueling boom
}

\author{
Yaohong $\mathrm{Qu}^{*}$, Xue $\mathrm{Mu}$, Zhuoya Wang, and Bing Xiao \\ Northwestern Polytechnical University, Xi'an, Shaanxi, China
}

\begin{abstract}
This paper studies on the refueling boom aerodynamic characteristics and proposes an elastic suppression method for the refueling boom with the property of large length-diameter ratio. In order to obtain the accurate frequency response, modal analysis for the refueling boom is conducted based on ANSYS MODAL. Then the dynamics and kinematic equations of the elastic refueling boom are deduced, which contain the frequency parameter. To complete the equations, the aerodynamic performance is solved in ANSYS FLUENT. In addition, based on the mixed $H_{2} / H_{\infty}$ theory, an optimal PID controller is proposed to suppress the elastic deformation. Furthermore, the parameters of the PID controller are optimized with the differential evolution algorithm. Simulation shows that the different elastic mode has different effect on the control of the refueling boom. The elastic deformation is suppressed obviously for each mode.
\end{abstract}

Keywords: refueling boom; aerodynamic analysis; modal analysis; elastic suppression; mixed optimal PID controller

\section{Introduction}

In recent years, modeling and controlling of the refueling boom have been widely attention and studied. In [1], a preliminary aerodynamic model of the refueling boom under the background of EU FP7 RECREATE is putting forward. In order to improve the model, the hinge joint is taken into account in [2-3], but the estimation of aerodynamic parameters is rough. In [4], the finite element analysis is used to obtain the model of the structural force of the refueling boom. Furthermore, several dynamic models of the refueling boom are considered, which are closer to the actual conditions. One of them is a mathematic refueling boom model with changing mass caused by the fuel transfer [5-6]. The other introduces the boom extension of the dynamic model, where the static model and moving model are established [7].

It can be seen from the researches that the elastic deformation of the refueling boom is not considered. Nonetheless, extensive studies on the elastic aircraft have been made. Waszak and Schmidt propose the nonlinear equations of motion for an elastic aircraft based on the Lagrange equation and the principle of virtual work [8]. In [9], Schmidt and Raney extend this work and demonstrated the importance of coupled flight dynamics and structural dynamics [9]. On basis of it, Shearer develops an integrated flight dynamic model for a representative high altitude long endurance vehicle [10]. In order to simplify the model without changing performance, a reduced-order aeroelastic model is proposed [9].
In general, the researches to the elastic problem, especially the elastic refueling boom, are not mature enough. In this paper, the modeling and control of elastic refueling boom are investigated. For this purpose, we analyse the modes of elastic refueling boom based on ANSYS MODAL. Then, based on the simulation of the aerodynamic parameters in FLUENT, the model of the refueling boom is improved. Further, using the parameters, we deduced the dynamics and kinematic equations of the elastic refueling boom. On basis of it, the optimal PID controller to control the attitude and suppress the elastic deformation of the refueling boom is proposed. Simulation results verify the effectiveness of the proposed method.

\section{Modal analysis of the refueling boom}

\subsection{Theoretical analyses of the mode}

As the vibration characteristic of the object structure itself, the mode possesses the following parameters, inherent frequency, mode shapes and damping ratio. These parameters are mainly obtained by two approaches, mathematical method and experiment. This paper takes the method of mathematic to deduce the parameter characteristics of the mode.

Suppose there are $n$ freedoms to the refueling boom, whose vibration differential equation is

$$
M \ddot{X}+C \dot{X}+K X=F
$$

where $M, C, K$ and $F$ is the mass matrix, damping matrix, stiffness matrix and the external force matrix of

\footnotetext{
* Corresponding author: qyh0809@,126.com
} 
the elastic refueling boom respectively. $\ddot{X}, \dot{X}$ and $X$ is respectively the acceleration vector matrix, the velocity vector matrix and the displacement vector matrix of the elastic refueling boom.

Assume the external force is zero. According to Fourier transform

$$
\left(K-\omega^{2} M\right) X(\omega)=0
$$

Define a set of generalized coordinates

$$
\left\{q_{1}(\omega) \quad q_{2}(\omega) \quad \ldots \quad q_{n}(\omega)\right\}
$$

Then $X(\omega)$ can be represented as follow.

$$
X(\omega)=\left[\begin{array}{llllll}
\phi_{1} & \phi_{2} & \ldots & \phi_{r} & \ldots & \phi_{n}
\end{array}\right]\left[\begin{array}{c}
q_{1}(\omega) \\
q_{2}(\omega) \\
\vdots \\
q_{n}(\omega)
\end{array}\right]=\Phi Q
$$

where $\phi_{r}=\left[\begin{array}{llll}\varphi_{1 r} & \varphi_{2 r} & \ldots & \varphi_{n r}\end{array}\right]^{T}$ is the $r$ th vibration mode of the elastic refueling boom, $\Phi$ is the modal matrix of the elastic refueling boom.

$$
\left(K-\omega^{2} M\right) \Phi=0
$$

Based on the solvable conditions of equation, characteristic equation Eq. (8) is deduced.

$$
\operatorname{det}\left|K-\omega^{2} M\right|=0
$$

Suppose the characteristic equation has no multiple root. We arrange $n$ positive different roots $\omega_{r}$, which solved by Eq. (8), in ascending order.

$$
0<\omega_{1}<\omega_{2}<\ldots<\omega_{r}<\ldots<\omega_{n}
$$

where $\omega_{r}$ is the $r$ th inherent frequency of the refueling boom. For each inherent frequency, the corresponding vibration mode can be solved.

In theory, infinite number of eigenvalues and corresponding eigenvectors can be obtained in the solving processes. Nevertheless, the eigenvalues and eigenvectors arranged in the first few modes play the major role to the actual vibration system.

\subsection{Modal analysis base on the software}

In term of the conclusion in previous section, there are infinite number of modes for the elastic refueling boom. With that, it's impossible to realize manual computation. Therefore, this paper utilizes several software to solve the elastic modal. The airfoil data of the winglet which installed on the refueling boom are generated in PROFILI. Then the winglet is generated by importing the airfoil data into CATIA and is assembled on the created three-dimensional model of refueling boom. The analysis of the elastic mode is carried out in ANSYS MODAL at last.

Based on the results of modal analysis in ANSYS, inherent frequencies of six lowest orders that corresponding to the first six order modes of refueling boom are obtained. Table 1 shows inherent frequencies of refueling boom.

Fig. 3 implies the deformation diagrams of the six lowest order vibration modes by ANSYS software. It shows that, for every mode, the vibration of refueling boom is violent. However, there are no vibration modes like this in real world. The reason is that different mode has different weight and the participation factor of high order mode is extremely small [10].

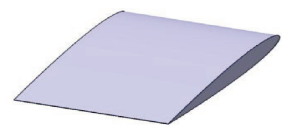

Figure. 1. The airfoil of the winglet generated in PROFILI.

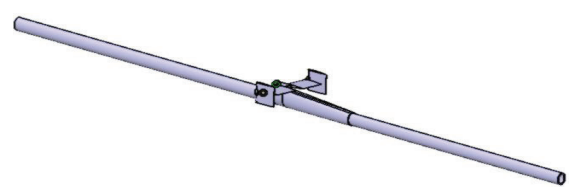

Figure. 2. Model of refueling boom created by CATIA.

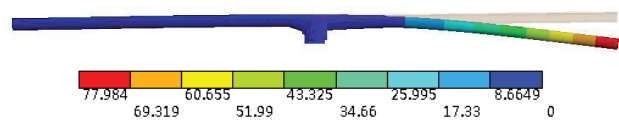

(a) The $1^{\text {st }}$ order vibration mode.

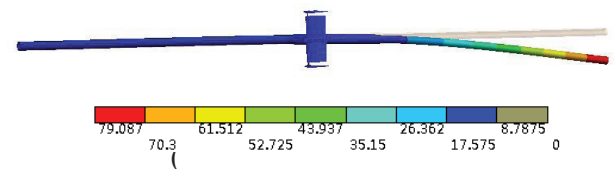

b) The $2^{\text {nd }}$ order vibration mode.

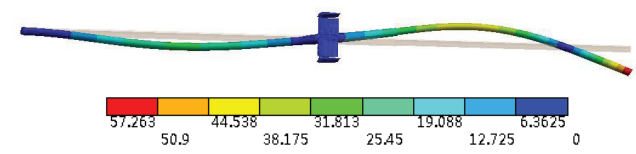

(c) The $3^{\text {rd }}$ order vibration mode.

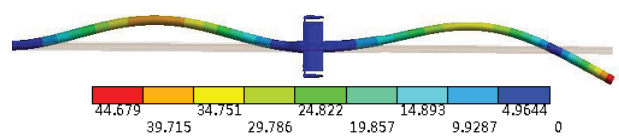

(d) The $4^{\text {th }}$ order vibration mode.

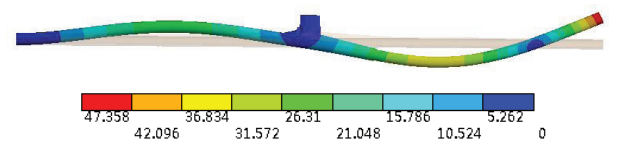

(e) The $5^{\text {th }}$ order vibration mode.

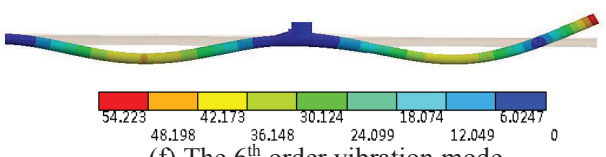

(f) The $6^{\text {th }}$ order vibration mode.

Figure. 3. The vibration modes of six lowest orders.

\subsection{Simulation of aerodynamic parameters}

Take the first mode of the elastic refueling boom as an example. The first order deformation diagram generated by MODAL is exported as a file with stp format. Then import the file to the ANSYS ICEM. After meshing the refueling boom and the fluid area of the atmospheric environment based on ICEM, the simulation of aerodynamic parameters is proceeded.

To acquire the aerodynamic force generated by deformed refueling boom, k-epsilon turbulence model is chosen as the flow field model of the atmospheric 
environment. Suppose the atmospheric environment surrounding the refueling boom is compressible ideal gas, the velocity of the airflow is $0.75 \mathrm{Mach}$ and the angle of attack $\alpha$ changes from $0^{\circ}$ to $16^{\circ}$ in $2^{\circ}$ increments. Then, the aerodynamic parameters are obtained. Table 2 to Table 4 show the coefficients of fitted curves to each mode. In Table 2 to Table 4, the general formulation is $y_{i}=a e^{b}$, where $i$ and $y$ denote the order of the mode and the fitted curve, respectively. In each aerodynamic parameter calculation, the iterative convergence condition of the value must satisfy the requirement. Fig. 4 is the residual convergence diagram of aerodynamic parameters in once iterative process.

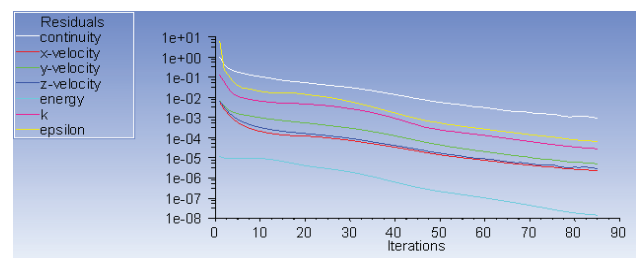

Figure. 4. The residual convergence diagram of aerodynamic parameters in once iterative process.

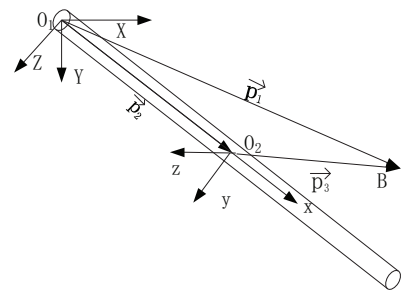

Figure. 5. Schematic diagram of refueling boom.

Table 1. Six lowest inherent frequencies of refueling boom

\begin{tabular}{|c|c|c|c|c|c|c|}
\hline Mode & 1 & 2 & 3 & 4 & 5 & 6 \\
\hline $\begin{array}{c}\text { Inherent } \\
\text { frequenc } \\
\mathrm{y}\end{array}$ & $\begin{array}{c}0.887 \\
8\end{array}$ & $\begin{array}{c}1.026 \\
4\end{array}$ & $\begin{array}{c}3.573 \\
8\end{array}$ & $\begin{array}{c}5.632 \\
7\end{array}$ & $\begin{array}{c}8.468 \\
3\end{array}$ & $\begin{array}{c}10.268 \\
1\end{array}$ \\
\hline
\end{tabular}

Table 2. Coefficients to each mode about lift moment

\begin{tabular}{|c|c|c|}
\hline \multirow{2}{*}{ Order } & \multicolumn{2}{|c|}{ Lift Coefficients } \\
\cline { 2 - 3 } & $a$ & $b$ \\
\hline 1 & 0.1134 & $0.0985 \alpha$ \\
\hline 2 & 0.1236 & $0.0985 \alpha$ \\
\hline 3 & 0.1371 & $0.0985 \alpha$ \\
\hline 4 & 0.1449 & $0.0985 \alpha$ \\
\hline 5 & 0.1501 & $0.0985 \alpha$ \\
\hline 6 & 0.1601 & $0.0985 \alpha$ \\
\hline
\end{tabular}

Table 3. Coefficients to each mode about resistance moment

\begin{tabular}{|c|c|c|}
\hline \multirow{2}{*}{ Order } & \multicolumn{2}{|c|}{ Resistance Coefficients } \\
\cline { 2 - 3 } & $a$ & $b$ \\
\hline 1 & 0.5910 & $-[(\alpha-15.16) / 7.724]^{2}$ \\
\hline 2 & 0.5330 & $-[(\alpha-15) / 7.724]^{2}$ \\
\hline 3 & 0.4481 & $-[(\alpha-14.78) / 7.724]^{2}$ \\
\hline 4 & 0.3999 & $-[(\alpha-16) / 7.724]^{2}$ \\
\hline 5 & 0.2223 & $-[(\alpha-15.99) / 7.724]^{2}$ \\
\hline 6 & 0.1123 & $-[(\alpha-13.56) / 7.724]^{2}$ \\
\hline
\end{tabular}

Table 4. Coefficients to each mode about pitch moment

\begin{tabular}{|c|c|c|}
\hline \multirow{2}{*}{ Order } & \multicolumn{2}{|c|}{ Pitch Coefficients } \\
\cline { 2 - 3 } & $a$ & $b$ \\
\hline 1 & 0.02666 & $-[(\alpha-0.2991) / 7.337]^{2}$ \\
\hline 2 & 0.02899 & $-[(\alpha-0.3695) / 7.595]^{2}$ \\
\hline 3 & 0.03536 & $-[(\alpha-0.4991) / 7.837]^{2}$ \\
\hline 4 & 0.03676 & $-[(\alpha-0.5991) / 8.137]^{2}$ \\
\hline 5 & 0.03996 & $-[(\alpha-0.6991) / 8.537]^{2}$ \\
\hline 6 & 0.04529 & $-[(\alpha-0.7991) / 8.637]^{2}$ \\
\hline
\end{tabular}

\section{Problem formulation}

\subsection{The refueling boom with elastic deformation}

The body coordinate frame $\left(O_{1} X Y Z\right)$ of the tanker and the coordinate frame of refueling boom $\left(\mathrm{O}_{2} x y z\right)$ are built in Fig. 5, where $O_{1}$ is at the universal joint which between tanker and refueling boom, $\mathrm{O}_{2}$ is the center of mass for refueling boom, where $\vec{p}_{1}=\vec{p}_{2}+\vec{p}_{3}$.

There is angular velocity $\vec{\omega}$ between two coordinate frames $O_{1} X Y Z$ and $O_{2} x y z$. The kinetic energy of the elastic refueling boom can be represented

$$
\begin{aligned}
& E_{k}=\frac{1}{2} \int_{V}\left\{\frac{d \vec{p}_{2}}{d t} \frac{d \vec{p}_{2}}{d t}+\frac{\delta \vec{p}_{3}}{\delta t} \frac{\delta \vec{p}_{3}}{\delta t}+\left(\vec{\omega} \times \vec{p}_{3}\right)\left(\vec{\omega} \times \vec{p}_{3}\right)\right. \\
& \left.+2 \frac{d \vec{p}_{2}}{d t} \frac{\delta \vec{p}_{3}}{\delta t}+2 \frac{d \vec{p}_{2}}{d t}\left(\vec{\omega} \times \vec{p}_{3}\right)+2 \frac{\delta \vec{p}_{3}}{\delta t}\left(\vec{\omega} \times \vec{p}_{3}\right)\right\} \rho d V
\end{aligned}
$$

where $\rho, V, \rho d V$ mean the density of refueling boom, the volume of refueling boom, and the elementary mass. $\delta / \delta(\cdot)$ represents the time derivative of the coordinate frame for refueling boom.

According to the theory of mean axis, the kinetic energy and the gravitational potential energy of the elastic refueling boom can be rewritten as

$$
\begin{gathered}
E_{k}=\frac{1}{2} M \frac{d \vec{p}_{2}}{d t} \frac{d \vec{p}_{2}}{d t}+\frac{1}{2} \int_{V} \frac{\delta \vec{p}_{3}}{\delta t} \frac{\delta \vec{p}_{3}}{\delta t} \rho d V+\frac{1}{2} \omega^{T}[I] \omega \\
E_{p g}=-\vec{p}_{2} M g
\end{gathered}
$$

where $M$ and $I$ are respectively the mass of the refueling boom and the inertia tensor matrix.

$$
\begin{gathered}
E_{p e}=\frac{1}{2} \sum_{i=1}^{\infty} \omega_{i}^{2} q_{i}^{2} M_{i} \\
E_{k}=\frac{1}{2} M \frac{d \vec{p}_{2}}{d t} \frac{d \vec{p}_{2}}{d t}+\frac{1}{2} \sum_{i=1}^{\infty} M_{i} \dot{q}_{i}^{2}+\frac{1}{2} \omega^{T}[I] \omega, M_{i}=\int_{V} \vec{\phi}_{i} \vec{\phi}_{i} \rho d V
\end{gathered}
$$

Based on the facts that the deformation quantity is extremely small essentially and the modes are orthogonal, the final forms of the elastic potential energy and the kinetic energy are presented as Eq. (15), (16), where $q_{i}$ is the generalized coordinate, and $\omega$ means the modal frequency.

By the principle of dynamics, $\vec{p}_{2}, d \vec{p}_{2} / d t$ and $\vec{\omega}$ can be defined as follows

$$
\begin{gathered}
\vec{p}_{2}=x \vec{i}+y \vec{j}+z \vec{k} \\
d \vec{p}_{2} / d t=u \vec{i}+v \vec{j}+w \vec{k} \\
\vec{\omega}=p \vec{i}+q \vec{j}+r \vec{k}
\end{gathered}
$$


where $p=\dot{\phi}, q=\dot{\theta} \cos \phi, r=-\dot{\theta} \sin \phi, x=l \cos \theta, y=l \sin \theta$, $z=0 . \theta$ represents the roll angle and $\phi$ denotes the pitch angle. We defined $l$ as the distance from the universal joint to the center of mass for refueling boom.

By the formulations of $E_{k}$ and $E_{p g}$, it can be deduced as follows

$$
\begin{gathered}
E_{k}=\frac{1}{2} M\left(u^{2}+v^{2}+w^{2}\right)+\frac{1}{2} \sum_{i=1}^{\infty} M_{i} \dot{q}_{i}^{2}+\frac{1}{2}\left[\begin{array}{lll}
p & q & r
\end{array}\right]\left[\begin{array}{lll}
I
\end{array}\right]\left[\begin{array}{lll}
p & q & r
\end{array}\right]^{T} \\
E_{p g}=-M g(-x \sin \theta+y \sin \phi \cos \theta+z \cos \phi \cos \theta)
\end{gathered}
$$

The following equations (18-25) can be deduced by combining Lagrange equations with the formulations of virtual work

$$
\begin{gathered}
M(\dot{u}-v r+w q+g \sin \theta)=X \\
M(\dot{v}-w p+u r-g \sin \phi \cos \theta)=Y \\
M(\dot{w}-u q+v p-g \cos \phi \cos \theta)=Z \\
\dot{p} I_{x}-\dot{r} I_{x z}+q r\left(I_{z}-I_{y}\right)-p q I_{x z}=M_{x}
\end{gathered}
$$

$\dot{q}(\cos \phi-p \sin \phi) I_{y}+(p r \cos \phi-\dot{r} \sin \phi) I_{z}+\left(\dot{p} \sin \phi+p^{2} \cos \phi\right) I_{x z}$

$+M g \sin \phi(x \sin \phi \cos \theta+y \sin \theta)+M \cos \phi(v p x-u p x+w q z+v r z$

$-w q z-w r y)+M \sin \phi(\dot{u} y-v r y+w p y-\dot{v} x-u r x+v q z)=\cos \phi M_{y}$

$$
\begin{gathered}
M_{i} \ddot{q}_{i}+M_{i} \omega_{i}{ }^{2} q_{i}=Q_{q i} \\
M_{x}=l_{1} Q s_{1} C_{l \delta_{e}} \delta_{r}+l Q s \sum_{i=1}^{\infty} C_{l q_{i}} q_{i} \\
M_{y}=l_{1} Q s_{2} C_{m \delta_{r}} \delta_{e}+l Q s \sum_{i=1}^{\infty} C_{m q_{i}} q_{i}
\end{gathered}
$$

where $M_{x}$ and $M_{y}$ are the pitch moment and the roll moment of refueling boom, respectively. $C_{m \delta_{r}}, C_{l q_{i}}, C_{l \delta_{e}}$ and $C_{m q_{i}}$ are relative aerodynamic coefficients of refueling boom, where $C_{l \delta_{e}}$ and $C_{m \delta_{r}}$ are effected by the deflection of the control surface $\delta_{e}$ and $\delta_{r}$, respectively. $C_{l q_{i}}, C_{m q_{i}}$ represent the aerodynamic parameters of the boom under the $i$ th mode. We acquire these parameters by fitted curves. $s, s_{1}, s_{2}$ are respectively the reference area of the refueling boom, the elevator area, the rudder area. $l_{1}$ represents the distance from the top of the refueling boom to the winglet. $Q_{q i}$ can be found in [8].

\section{Controller design}

\subsection{The mixed $\boldsymbol{H}_{2} / \boldsymbol{H}_{\infty}$ optimal PID controller with the differential evolution algorithm}

In this section, on the basis of elastic refueling boom model after linearization, a controller will be developed for the elastic suppression.

There are 16 state variables in the proposed model. For convenience of simulation and calculation, model reduction is implemented using balanced realization method [11] and Hankel singular value method [12]. The reduction model is Eq. (26).

$$
\begin{aligned}
& \dot{\mathbf{x}}_{1}=\bar{A}_{I I} \overline{\mathbf{x}}_{1}+\overline{\boldsymbol{B}}_{1} \mathbf{u} \\
& \mathbf{y}=\overline{\boldsymbol{C}}_{I} \overline{\mathbf{x}}_{1}
\end{aligned}
$$

where $\quad \overline{\mathbf{x}}_{1}=\left[\begin{array}{llllll}\theta & \dot{\theta} & \phi & \dot{\phi} & q_{i} & \dot{q}_{i}\end{array}\right]^{T}(i=1,2,3,4,5,6)$ is state variable. $\mathbf{u}=\left[\begin{array}{ll}\delta_{\mathrm{e}} & \delta_{\mathrm{r}}\end{array}\right]^{\mathrm{T}}$ is input, and $\mathbf{y}=\left[\begin{array}{ll}\theta & \phi\end{array}\right]^{T}$ is output.

The optimal PID controller for the mixed $\mathrm{H}_{2} / \mathrm{H}_{\infty}$ with the differential evolution algorithm is a method to improve the robustness of PID controller.

$$
\begin{gathered}
K(s)=k_{p}+\frac{k_{i}}{s}+k_{d} s \\
S(s)=(1+K(s) F(s))^{-1} \\
T(s)=K(s) F(s)(1+K(s) F(s))^{-1}
\end{gathered}
$$

$W_{1}(s), W_{2}(s)$ should meet the conditions

$$
\begin{aligned}
& \bar{\sigma}[S(j \omega)] \leq \bar{\sigma}\left[W_{S}^{-1}(j \omega)\right] \\
& \bar{\sigma}[T(j \omega)]<\bar{\sigma}\left[W_{T}^{-1}(j \omega)\right]
\end{aligned}
$$

where $F(s), K(s)$ are respectively the controlled object and the PID controller. $W_{1}(s), W_{2}(s)$ are the weight functions of sensitivity $S(s)$ and complementary sensitivity $T(s)$ respectively.

The singular values of $S(s), T(s)$ are expected to reach the minimum. For $S(s)$ and $T(s)$, there are two optimization indicators.

$$
\begin{aligned}
& J_{S}=\left\|W_{s}(s) S(s)\right\|_{a} \\
& J_{T}=\left\|W_{T}(s) T(s)\right\|_{a}
\end{aligned}
$$

The optimization indicator is obtained further

$$
\begin{gathered}
J_{\infty}=\left(J_{T}^{2}+J_{S}^{2}\right)^{1 / 2} \\
J=J_{\infty}+J_{2}
\end{gathered}
$$

where $J$ is the comprehensive optimization indicator.

The differential evolution theory is used to minimize the optimization indicator to optimize the three parameters of the optimal PID controller. Furthermore, we obtain the mixed $H_{2} / H_{\infty}$ optimal PID controller. Suppose the individual $\mathbf{x}=\left[\begin{array}{lll}k_{p} & k_{i} & k_{d}\end{array}\right]$, whose initialization is $\mathbf{x}_{\mathbf{i}}=\left(x_{i 1}, x_{i 2}, x_{i 3}\right)$, where $i \in[1,50], x_{i} \in[0,20]$.

The iteration begins:

Step 1: Get variation individuals

$$
v_{i}=x_{i}+F\left(x_{r 1}-x_{r 2}\right)
$$

where $F=0.5$.

Step 2: Obtain hybrid individuals

$$
u_{i j}=\left\{\begin{array}{cc}
v_{i j} & \text { rand }[0,1] \leq P_{r}, \quad \text { or } j=j_{\text {rand }} \\
x_{i j} & \text { else }
\end{array}\right.
$$

where $P_{r}=0.3$.

Step 3: Select the next generation of individuals

$$
x_{i}^{g+1}= \begin{cases}u_{i} & f\left(u_{i}\right)<f\left(x_{i}^{g}\right) \\ x_{i}^{g} & f\left(u_{i}\right) \geq f\left(x_{i}^{g}\right)\end{cases}
$$

where $f(\cdot)$ is the optimizing index for the optimal PID controller.

\subsection{Simulink the optimal controller designed}

On the basis of the previous section, select the weight function of sensitivity and complementary sensitivity according to the principle of weight function selection. 


$$
\begin{gathered}
W_{1}(s)=\frac{200}{175 s+1} \\
W_{2}(s)=\frac{0.2146 s+0.0011}{0.45 s+1}
\end{gathered}
$$

After 100 iterations of the differential evolution algorithm, the optimal PID controller parameters with mixed $H_{2} / H_{\infty}$ are $k_{p}=20.356, k_{i}=10.928, k_{d}=1.063$.

Assume the flight velocity of the tanker is $200 \mathrm{~m} / \mathrm{s}$. Then, we simulate the proposed optimal PID controller. The simulation results are shown in Fig. 6.

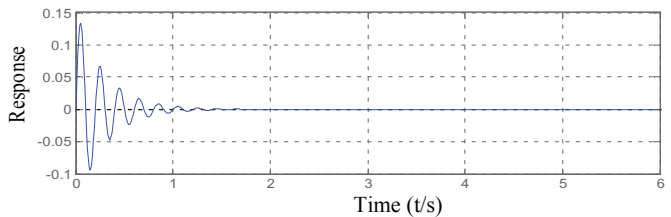

(a) Response of the $1^{\text {st }}$ order mode.

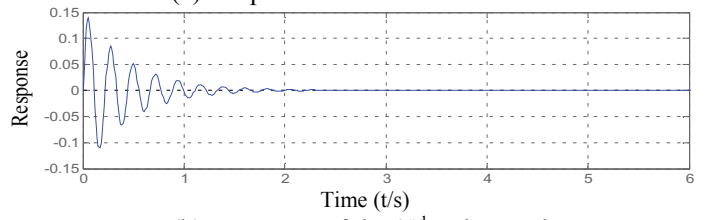

(b) Response of the $2^{\text {nd }}$ order mode.

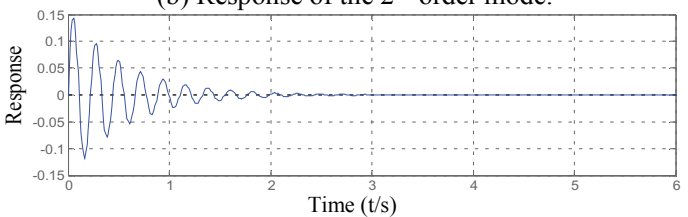

(c) Response of the $3^{\text {rd }}$ order mode.

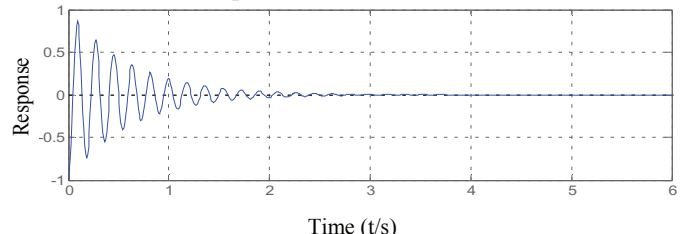

(d) Response of the $4^{\text {th }}$ order mode.

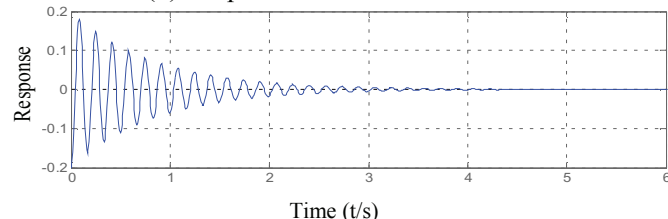

(e) Response of the $5^{\text {th }}$ order mode.

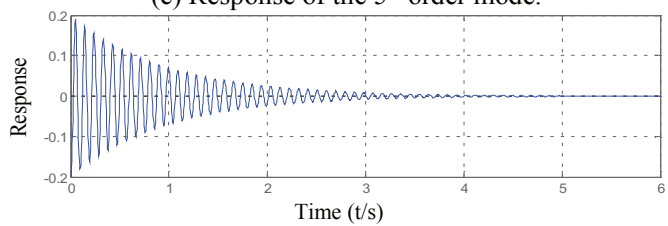

(f) Response of the $6^{\text {th }}$ order mode.

Figure. 6. Response curves at the objective $\left(30^{\circ}, 0^{\circ}\right)$ to $\left(32^{\circ}, 0^{\circ}\right)$.

\section{Conclusion}

In this paper, the modal analysis and aerodynamic analysis of elastic refueling boom have been developed by ANSYS. Through the analysis of the elastic refueling boom, the accurate frequency response and aerodynamic characteristics are obtained. Further, a proposed model is deduced and with that an optimal PID controller to suppress the elastic deformation is proposed. The validity of the proposed controller is verified by simulation. That is, the elastic deformation of each mode is also obviously suppressed.

\section{Acknowledgements}

This work is supported by the National Natural Science Foundation of China (No. 61473229).

\section{References}

1. Manfriani L, Righi M. Aerodynamic modeling of a refueling boom. 29th Congress of the International Council of the Aeronautical Sciences, ICAS 2014.

2. Chang L, Jia YM. Analog strategy of a receiver aircraft tested for boom and receptacle refueling. Chinese Control Conference. 2015,5857-5862.

3. Yuan DL, Zhao CX, Xiao ZB. Research on path planning of manipulator for aerial boom refueling test system. Applied Mechanics and Materials, 2014, 538:159-162.

4. Yang Q, Li C, Ji Q. Application of non-cancellation decoupling in boom refueling control. International Conference on Mechatronics and Automation, 2009:4203-4207.

5. David I.L, Bernard F. Visual comparisons of selected shores affected by the Prestige oil spill, Northern Spain 2002-2004. 2005 International Oil Spill Conference, 2005:10514-10517.

6. Zhu Z.H, Meguid S.A. Elastodynamic analysis of aerial refueling hose using curved beam element. AIAA Journal, 44(6): 1317-1324.

7. Yang C, Yang Y, Lu Y. Modeling and simulation for refueling boom and receiver in coupled mode. Transactions of Nanjing University of Aeronautics and Astronautics, 2017, 34(2):143-151.

8. Waszak M.R, Schmidt D.K. Flight dynamics of aeroelastic vehicles. Journal of Aircraft, 1988, 25(6): 563-571.

9. Baghdadi N, Lowenberg M.H, Isikveren A.T. Analysis of flexible aircraft dynamics using bifurcation methods. Journal of Guidance, Control, and Dynamics, 2011, 34: 795-809.

10. Skogestad S, Postlethwaite I. Multivariable feedback control. New York: Wiley, 2005.

11. Wortelboer P, Odstveen H.V. Model reduction guided by Hankel singular value intervals. Identification, Model and Control, 1990, 1:17-26.

12. Ho SJ, Ho SY, Hung MH, Shu LS, Hung HL. Designing structure-specified mixed optimal $\mathrm{H}_{2} / \mathrm{H}_{\infty}$ controllers using an intelligent genetic algorithm IGA. IEEE Trans on Control Systems Technology, 2005, 13(6): 1119- 1124 . 